\title{
Prediction of Spark Kernel Development in Constant Volume Combustion
}

\author{
M. T. LIM \\ Tech. Res. Inst., Hyundai Precision and Industry Co. Ltd., Seoul, South Korea
}

R. W. ANDERSON

Engine Research Dept., Research Staff, Ford Motor Co., Dearborn, MI 48121-2053

\section{S. ARPACI}

Dept. Mech. Engrg. and Applied Mech., University of Michigan, Ann Arbor, MI 48109

\begin{abstract}
Combustion initiation is studied in atmospheric pressure propane-air mixtures in a constant volume bomb with a high speed (10,000 fps) laser schlieren system. The spark current and voltage waveforms are simultaneously recorded for later model input. A phenomenological model for early flame kernel development is presented which accounts for the initial, breakdown generated, spark kernel and its subsequent growth. The kernel growth is initially controlled by the breakdown process and the subsequent electrical power input. A new, spark power induced, mass entrainment term is shown to model this initially rapid volume increase adequately while later growth is mainly dominated by diffusion. Results and model comparisons are presented for the effects of power input, spark energy, and equivalence ratio.
\end{abstract}

\section{INTRODUCTION}

Improved fuel economy under the constraint of strict emission standards has become one of the most important goals in engine design since the oil crises in the 1970s. One of the promising solutions is fuel lean combustion. In an attempt to extend the lean ignition limit [1] and shorten the initial burn time to improve combustion stability, modifications to conventional ignition systems as well as new ignition devices are being sought and some are already implemented [2]. Although a complete understanding of spark ignition for even a quiescent mixture remains to be achieved, a qualitative understanding of the numerous factors affecting ignition is beginning to emerge.
There is an extensive amount of literature on spark ignition. However, because of its size, only the relevant aspects are summarized (see, for example, Lim [3] for a recent and extensive review). The literature demonstrates the time dependence of the ignition process [4-12]. One must consider not only how much energy is applied to the mixture, but the rate of application and the mode of discharge [4, 7-13]. Additionally, heat transfer to even thin electrodes is observed to be important $[5,6,13,14]$. Combustion reactions occur quite early after electrical breakdown of the gap and the initial flame kernel growth is shown to be very rapid $[11,12,15-19]$. One clearly desires to account for all of these processes in attempting to understand ignition, but is confronted with 
computational constraints if multidimensional modeling is pursued [20-25]. A phenomenological approach that predicts spark kernel development while retaining the essential ingredients can provide some useful insights into this complex process.

An ignition study which uses an engine [26] is generally limited by an inability to control precisely and measure the local mixture condition on a cycle-by-cycle basis. In addition, there are experimental difficulties in monitoring the kernel growth process-even though the desired goal is the development of an engine ignition model. Consequently, the present study is addressed to the understanding of spark ignition in the quiescent environment of a combustion bomb.

The experimental part of the study follows in Section II where the developing spark kernel in a propane-air mixture is investigated with a high speed laser schlieren system. The effects of varying power and energy from a typical inductive source are studied using mixtures of different equivalence ratios. Based upon the experimental findings, a phenomenological model for predicting the initial flame kernel development is proposed in Section III. Section IV contains a comparison of the model and experiment with related discussions while Section $V$ presents a summary of the findings and concluding remarks.

\section{EXPERIMENTAL INVESTIGATION}

A high speed photographic record provides a valuable history of the shape and size of a developing flame kernel. This type of information is essential for direct comparison with and validation of detailed models. Several photographic studies of ignition are available $[13,15-17,27$, 28]. None of these investigations, however, includes a temporal distribution of the spark energy with the dimensional measurement. A variation of the temporal distribution of the spark energy influences the minimum ignition energy as well as the rate of kernel growth [10, 29, 30]. Moreover, there is run-to-run variation in the spark discharge which also affects the shape and size of the resulting kernel. Single frame photographic techniques are utilized in all of the referenced experi- ments. Pictures are taken of different sparks and then patched together to simulate a complete history of one event with the assumption that the process is repeatable.

\section{Experimental Approach}

The experimental part of the present study provides some high speed movies of developing spark kernels in a constant volume bomb with the laser schlieren arrangement of Fig. 1. The system allows precise timing of the first and subsequent frames by switching the laser beam into the test section at predetermined times and durations with an acoustooptic modulator while the high speed camera is run in the streak mode. A shutter speed of $1 \mu \mathrm{s}$ and framing rate of $10,000 \mathrm{fps}$ is used for this investigation.

The bomb is charged with an atmospheric pressure, propane-air, mixture. The spark kernels are produced by a variable energy inductive ignition system with duration control. The discharge waveforms are simultaneously recorded for each run for computation of the gap power input and the breakdown energy (see Lim [3] for further details).

\section{Data Analysis}

The shape of a spark kernel obtained without special measures is in general neither regular nor fixed in time. The projected area of the kernel is measured in such cases to compute an equivalent radius which is often the radius of a sphere with the same projected area [22]. The equivalent radius is a representative dimension of the kernel, but may give an inadequate estimate of the volume or surface area because of the spherical symmetry assumed in the conversion.

The approach taken in this study assumes no global symmetry of the spark kernel. A projected boundary of the kernel is divided into small segments in the axial direction of the electrodes. A local cylindrical symmetry of each lamina about the initial spark path, which is found from the locus of the weak shock wave trace in the first picture frame, is assumed in order to compute its contribution to the total volume and surface area. The portions of the kernel boundary on each side 


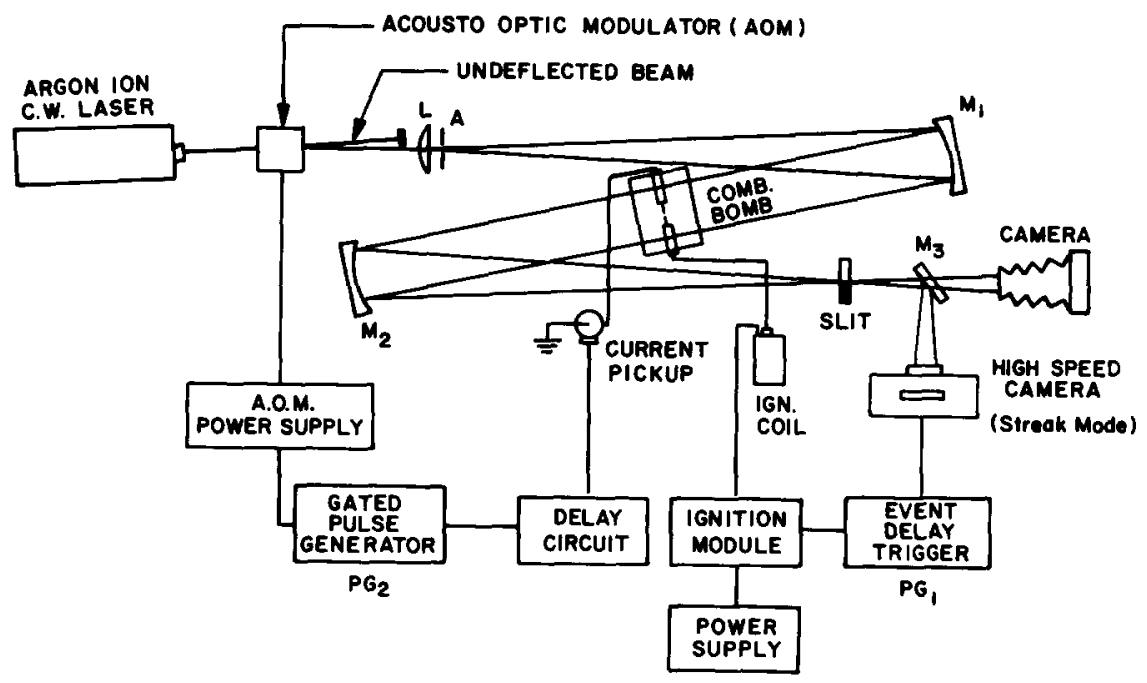

Fig. 1. Schematic of high speed schlieren system.

of the symmetry line are separately processed before the arithmetic averages are taken for the volume and surface area. The volume and surface area occupied by the electrodes within the kernel are subtracted. This method utilizes maximum available information from a projected kernel boundary but is not completely free from the inherent difficulties of computing 3-dimensional quantities from 2-dimensional photographs. Runs that exhibit an excessive degree of asymmetry in kernel shape are excluded to minimize this effect.

\section{Results and Discussion}

Schlieren photographs of a flame kernel developing in a propane-air mixture of equivalence ratio $\Phi=0.7$ are shown in Fig. 2. Numbers below the pictures represent the elapsed time in microseconds after spark initiation. The pictures are from a continuous recording of a single spark event. The first frame shows the high temperature kernel and the ellipsoidal trace of a weak shock wave that are generated by the breakdown event. The boundary of the kernel is wavy during the initial 400-700 $\mu \mathrm{s}$, but smoothes out in time. The wavy boundary of the kernel is observed in all of the cases studied and appears to be a result of the turbulence created by the blast during the breakdown of the gap. The electrodes restrain the expansion of the kernel in the axial direction, which causes the kernel shape to appear as a pinched ellipsoid at times later than 700-1000 $\mu \mathrm{s}$.

The volume and surface area computed with the method in the preceding section are cross-plotted on a $\log -\log$ scale in Fig. 3 for a wide variation of spark energy (2-55 mJ), equivalence ratio (0.01.0 ), and times greater than $5 \mu \mathrm{s}$. All the data fall very close to a straight line with a slope of 1.5 and a volume-area relationship which corresponds to a sphere while a slope of 2.0 corresponds to a long cylinder. The data indicate, therefore, that the spark kernels of this experiment are better modeled as spheres during the ignition phase. It should be noted that the sphericity of the kernels arises from the volumes and surface areas computed with the new method, while the projected area method assumes it from the beginning without giving consideration to its validation [3].

Since the relationship between the volume and surface area of all the spark kernels is that of a sphere in this experiment, only the equivalent spherical radii are presented as a function of time for different equivalence ratios and spark energies. It is not obvious, however, how closely the equivalent radius computed with the current method compares with that computed by the projected area method. One finds that they agree well at the early stages but diverge slightly from 


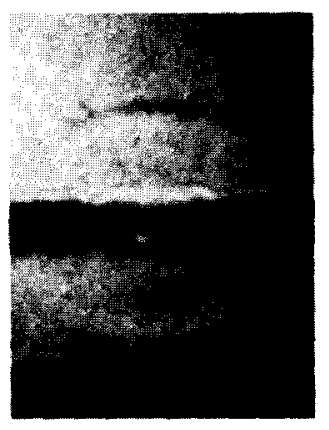

5

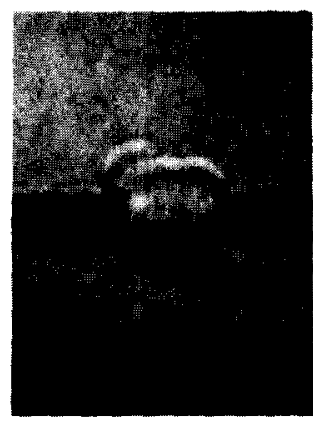

105

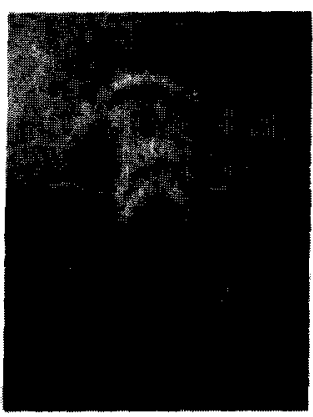

1005

705

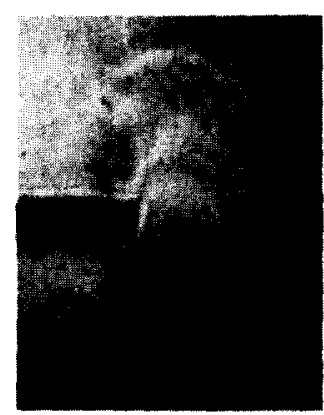

1905

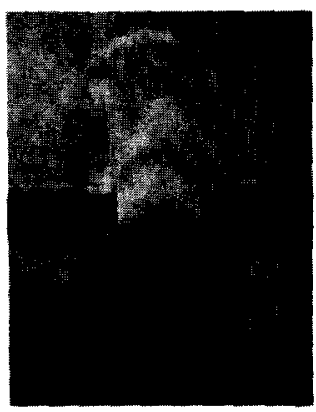

2405

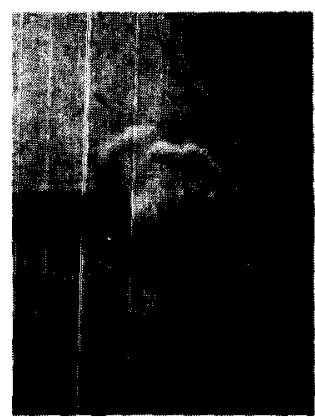

205

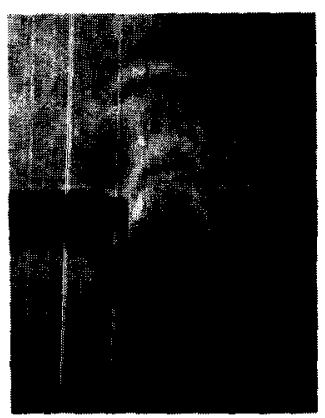

1305

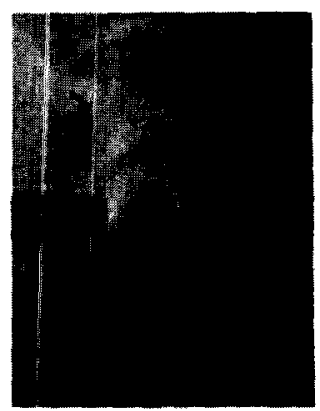

2905

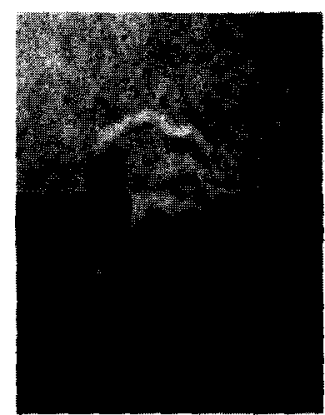

405

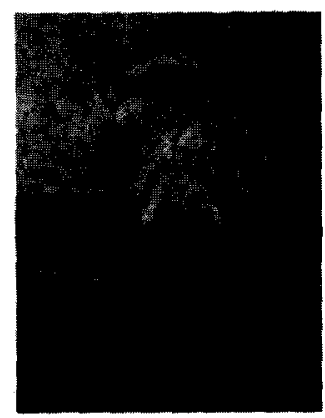

1605

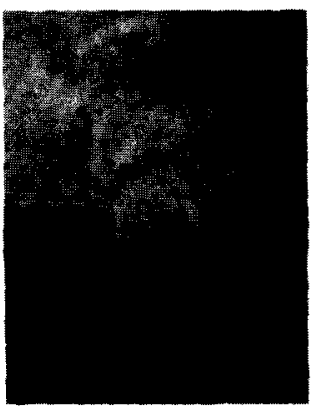

3905

Fig. 2. Typical example of schlieren pictures of a spark kernel. Propane-air mixture $(\boldsymbol{\Phi}=$ 0.7 ) at $1 \mathrm{~atm}$ and room temperature. Spark energy $=2.0 \mathrm{~mJ}$ and gap $=2.0 \mathrm{~mm}$. Numbers below pictures represent the elapsed time (in microseconds) after spark initiation. 


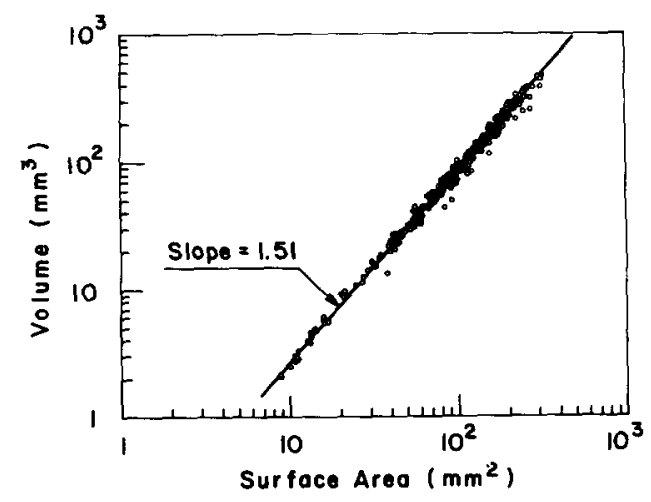

Fig. 3. Logarithmic plot of spark kernel volume versus surface area showing approximate spherical fit of data. Spark gap $=2$ $\mathrm{mm}$, spark energy $=2-54.8 \mathrm{~mJ}$, and $\Phi=0-1.0$.

each other as time progresses [3]. The smaller values from the projected area method come from the fact that the kernel does not actually grow in a completely spherical manner with finite size electrodes, but expands more in the transverse direction to the electrode axis and less in the axial direction. The current method gives more weight (proportional to the square of the local radius) to a projected area element far from the electrodes, while the projected area method does not.

Before presenting the modeling approach, some results previously found with this experiment $[3$, 31] and germane to the model are briefly summarized. The equivalent kernel radii of comparable sparks in air $(10.1 \mathrm{~mJ})$ and a propane-air mixture of equivalence ratio $0.7(11.5 \mathrm{~mJ})$ exhibit a difference in radii which grows larger in time as early as $5 \mu \mathrm{s}$ after the initiation of the discharge (first frame). This result contradicts Litchfield [27], who reports no difference in kernel size until the nonignition case separates from the ignition case but agrees with Akindele et al. [22]. The larger kernel in the combustible gas is attributed to chemical heat release from combustion reactions. Apparently the combustion reactions release enough heat to affect the kernel growth within a few microseconds. It appears to be important, therefore, to include this initial chemical heat release in models for spark kernel development. The growth rate of the kernel radius decreases monotonically from a very large value during the early phase toward an approximately constant rate at about $1 \mathrm{~ms}$. The estimated burning speed is equivalent to $17 \mathrm{~cm} / \mathrm{s}$ with respect to the unburned gas assuming a temperature of $1900 \mathrm{~K}$ (adiabatic flame temperature of the $\Phi=0.7$ mixture) inside the kernel. This value is lower than the normal flame velocity of $25 \mathrm{~cm} / \mathrm{s}$ from a laminar flame speed correlation by Lavoie [32] and is believed to be primarily due to curvature and heat transfer effects.

The effect of varying spark energy and duration on kernel development is also demonstrated in Refs. [3, 31]. The coil primary current is varied to obtain spark energies of approximately 2, 11.5, and $54.8 \mathrm{~mJ}$ with respective discharge durations of 264,810 , and $3730 \mu \mathrm{s}$. These ignition energies range from near the minimum level to an amount typical in current automotive engine use. The sizes of the spark kernels at $5 \mu \mathrm{s}$ are nearly identical to each other. This is anticipated because the total spark energy released by this time is close to the breakdown energy which is common for the three cases. After $100 \mu \mathrm{s}$, differences in radii appear and become larger in time. The kernel volume at $1500 \mu \mathrm{s}$ increases by about $84 \%$ when the spark energy increases from 2 to $11.5 \mathrm{~mJ}$ and an additional $40 \%$ for the $54.8 \mathrm{~mJ}$ case which has input $35 \mathrm{~mJ}$ of energy by this time. The additional spark energy after the first $11.5 \mathrm{~mJ}$ is therefore less effective in enhancing the flame kernel size. The interesting fact is that the considerable difference in the spark kernel size is caused by the additional energy over the minimum amount (about 1-2 $\mathrm{mJ}$ ) and is persistent at times when the spark energy is only a fraction of the combustion generated energy. Another interesting observation is that the flame kernel growth during the first 100 $\mu \mathrm{s}$ is about the same for the $11.5 \mathrm{~mJ}$ and $54.8 \mathrm{~mJ}$ cases which have comparable power inputs in this time frame but is distinctly less for the lower power $(2 \mathrm{~mJ})$ case. This power effect is shown by others $[10,11,19]$ to affect initial kernel development.

In an attempt to separate the effects of different power levels after the breakdown phase of the discharge, the primary current of the coil and spark discharge duration is adjusted so that sparks of comparable energy but different power are obtained. The higher power spark has a duration 


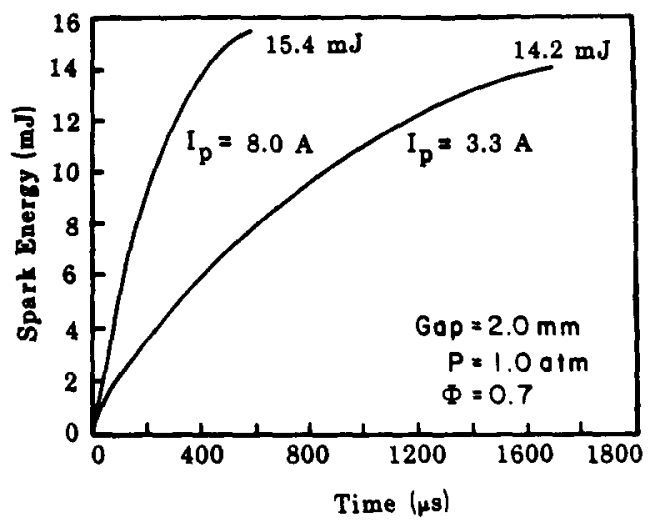

Fig. 4. Temporal distribution of the total energy for two sparks of comparable energy with different power levels (about 0.7 $1.5 \mathrm{~mJ}$ of breakdown energy excluded).

of $600 \mu \mathrm{s}$, while the lower power one is $1700 \mu \mathrm{s}$ as shown in Fig. 4. Because of run-to-run variation the higher power case turns out to have slightly more energy $(15.4 \mathrm{~mJ})$ than the lower one (14.2 $\mathrm{mJ})$. The measured kernel radii show a small difference in size that favors the lower power case immediately after spark initiation. This subtle difference persists throughout the time period of measurement (1500 $\mu \mathrm{s}$ ). A comparison of breakdown voltages reveals that the lower power $(3.3$ A) spark has roughly $100 \%$ more breakdown energy than the higher power $(8.0 \mathrm{~A})$ spark which is believed to be responsible for the initial size difference. The difference in radius is, however, statistically insignificant-especially after $400 \mu \mathrm{s}$ when the variation is larger than the difference itself. One concludes that a factor of about 2.5 in power level results in an insignificant difference in flame kernel size at the conditions employed in this study.

The change in equivalent kernel radius due to comparable sparks in stoichiometric and $\Phi=0.7$ propane-air mixtures is shown in Fig. 5. The spark energy inputs $(\approx 12.4$ and $11.5 \mathrm{~mJ}$, respectively, at $850 \mu$ s duration) are within experimental variation of each other. A small difference in the size of the kernel develops early and becomes larger as time progresses. This must be a result of the higher flame speed in addition to the greater combustion heat associated with the stoichiometric mixture. The expansion speed for the stoichiometric mixture is estimated at about $195 \mathrm{~cm} / \mathrm{s}$ which

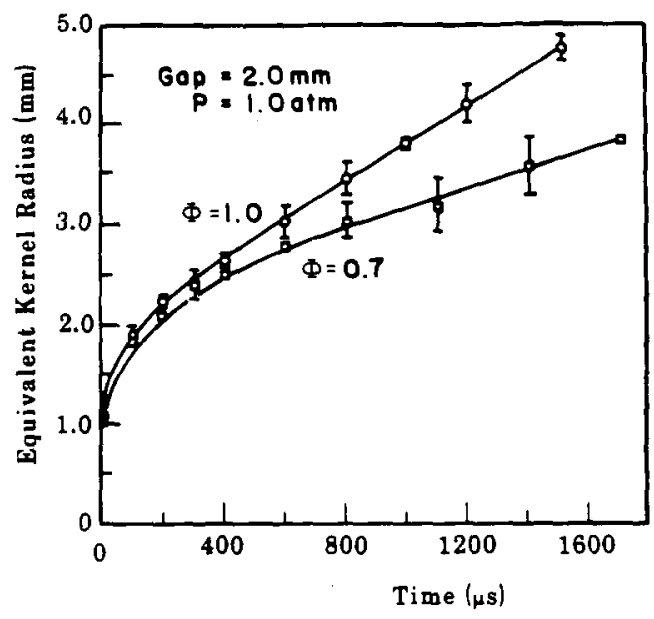

Fig. 5. Effects of equivalence ratio on development of initial flame kernels in lean propane-air mixtures at room temperature. The spark energy is $12.4 \mathrm{~mJ}$ for the $\Phi=1.0$ and $11.5 \mathrm{~mJ}$ for the $\Phi=0.7$ case.

corresponds to a burning speed of $26 \mathrm{~cm} / \mathrm{s}$ if the kernel temperature is that of an adiabatic flame. This burning speed is lower than the predicted steady value of $38.3 \mathrm{~cm} / \mathrm{s}$ for the mixture [32] and follows the earlier observation with the $\Phi=0.7$ mixture.

\section{A MODEL FOR FLAME KERNEL GROWTH}

There are a number of phenomenological models for prediction of the minimum ignition energy $[4$, $29,30,33-38]$. These models are lumped in space as well as in time. They provide a rough estimate of the minimum spark energy for a given mixture when a quenching distance gap is maintained between the electrodes. This is quite satisfactory for safety problems in accidental fire or explosion, but insufficient for IC engine applications where gap sizes, energy levels, and mixture conditions vary considerably from model assumptions. Furthermore, in addition to the question of ignition, it is also desired to know how fast the flame kernel grows, what will be the effect of excess spark energy over the minimum amount, and how the temporal distribution of the spark energy affects the process.

A phenomenological model is developed in this section which includes the early blast wave, later 
thermal diffusion characteristics, and some effect of the electrodes. The spark kernel is spatially lumped, but temporally varies in order to examine the effects of different distributions of the power input. The model attempts to simulate the developing spark kernels observed in the experimental part of the study.

The experimentally measured spark kernel radii appear to show that the kernel development is due to a diffusive process within a few microseconds after the breakdown event. This comes from the observation that the temporal variation of the kernel radius resembles a geometric parabola (see Fig. 5). The initial growth after spark initiation appears to be faster than by diffusion alone. As a result, the kernel appears to start from a finite size at time zero. This is a consequence of the blast wave phase which precedes the diffusional phase and is evidenced by the decaying shock wave observed during the first few microseconds (Fig. 2).

The spark kernel development appears to go through at least two different time domains: a short period of blast wave and a relatively long period of diffusion. A corresponding distinction is also observed from a distribution of spark power in time. Measurements of the initial breakdown phase [3] indicate that the power level difference between the first $20 \mathrm{~ns}$ and the rest of the discharge duration is 2-3 orders of magnitude. The high power level at the beginning (breakdown) is believed to form a small scale blast wave. The shock front of the blast wave rapidly decays to an acoustic wave with a time scale which is determined by the power input as well as the ambient gas condition. A gradual transition from the blast wave to the relatively slow diffusion phase takes place during this decay period (i.e., a few microseconds). Further growth of the initial spark kernel (hot core left by the blast) is mainly due to thermal diffusion when the subsequent electrical energy input occurs at low power.

\section{Blast Wave Phase}

Only a small fraction of the literature on blast waves is summarized here (see, for example, Lim [3] for a complete review). The blast wave problem originally formulated by Taylor [39, 40] and Sedov [41] has been extensively investigated with various solution techniques. Under the assumption of a strong shock front, a similarity solution for a blast wave formed by an instantaneous energy input is available. The assumption of a strong shock front is somewhat relaxed by the perturbation method. Approximate solutions are possible for further relaxation of the strong shock limitation at the cost of some accuracy. Exact numerical solutions to the formulation are also available for the entire lifetime of the blast wave. All of these solution techniques depend to some degree on numerical integration. The results are given in terms of dimensionless time and space coordinates, eliminating the need for further computations for parametric variations.

Because the initial dominance of the blast wave is relatively short in spark ignition, a blast wave solution from the literature is used as an initial condition for the ensuing diffusional phase. Since the initial discharge more closely approximates a line than a point source, Plooster's solution [42] for a cylindrical blast wave at the weak shock phase is chosen. He defines a dimensionless radius $\lambda$ and time $\tau$ as

$\lambda=\frac{r}{R_{0}}, \quad \tau=\frac{a t}{R_{0}}$,

where $R_{0}=\left(E_{0} / B \gamma p\right)^{1 / 2}$ is a characteristic length of the cylindrical blast due to an instantaneous energy release $E_{0}$ per unit length. The dimensionless parameter $B$ depends on the specific heat ratio of the gas and the shape of the blast $(B=3.94$ for $\gamma=1.4$ and cylindrical blast). The variables $t$ and $r$ are the temporal and spatial coordinates, and $p$ and $a$ are the pressure and the speed of sound of the ambient gas.

Plooster's results show that the gas velocity within the hot core is much less than sonic by $\tau \approx$ 0.19 and that the density and pressure jump across the shock front continue to decrease as the shock wave propagates outward. In the central region of the blast, the pressure initially decreases to a value lower than ambient which causes an inward gas flow at later times. After the pressure slowly recovers to the ambient value and the gas ceases to 
flow when $\tau \approx 3-4$, a hot (low density) region concentrates near the origin and remains relatively unchanged after $\tau \approx 1-2$. This region, which may be identified as the initial kernel in spark ignition, expands no further, as Adelman [30] points out. It is an expected result, however, because thermal diffusion is not included in the formulation of the problem. Thermal diffusion, which has been safely neglected during the early breakdown stage, now begins to become the dominating mechanism for the rest of the kernel growth.

With $1 \mathrm{~mJ}$ of input energy and normal temperature and pressure of the air, the nondimensional time $\tau=1.5$ in Plooster's blast wave solution corresponds to $3.6 \mu \mathrm{s}$. As noted above, the blast wave diminishes to an approximate acoustic wave by this time, leaving behind a hot core near the center that expands no further in size. This provides an estimate of the transition time from the blast wave to the diffusion wave.

A further confirmation of the transition time can be obtained from the fact that the fundamental difference between a blast wave and a diffusion wave is that the former is momentum-controlled while the latter is thermal energy-controlled. A dimensionless number that characterizes the relative importance of these waves can be derived from the ratio of kinetic energy and thermal conduction:

$$
\begin{aligned}
& {\left[\frac{\partial\left(\rho u^{2}\right)}{\partial t}\right] /\left[\frac{\partial}{\partial x}\left(\kappa \frac{\partial T}{\partial x}\right)\right]} \\
& \approx(\gamma-1)\left(\frac{u^{2}}{a^{2}}\right) \frac{L^{2}}{\alpha t} \approx \frac{(\gamma-1) M^{2}}{\text { Fo }},
\end{aligned}
$$

where $\alpha, a, \gamma$, and $L$ denote, respectively, the thermal diffusivity, isentropic speed of sound, ratio of the specific heats, and a characteristic length; $M$ is the Mach number and Fo the Fourier number.

At the transition from the blast wave to the diffusion wave, kinetic energy and diffusion are equally important. Then

$$
\frac{(\gamma-1) M^{2}}{F_{0}} \approx 1,
$$

which leads, in view of $u \approx L / t$, to

$t \approx\left[\left(\frac{\gamma-1}{\alpha a^{2}}\right)^{L^{4}}\right]^{1 / 3}$.

For a typical spark discharge in automotive engine use, the initial energy input is about $1 \mathrm{~mJ}$ and the kernel size is on the order of $1 \mathrm{~mm}$. For a kernel temperature of $2000 \mathrm{~K}$,

$t \approx 10^{-5} \mathrm{~s}$,

which is of the same order as the Plooster result and implies that the transition from the blast wave to the thermal diffusion wave occurs on the order of microseconds after spark initiation.

The ideal blast wave solution at $\tau=1.5$ is therefore used as an appropriate initial condition for the diffusion model. A kernel boundary is fixed at a radius $r_{\mathrm{k}}$, where the density assumes $90 \%$ of the undisturbed value $\rho_{0}$ and the density gradient becomes small. This is assumed to be the boundary that is detected in the schlieren photograph. The total mass inside the kernel is found from an integration of the density profile in Ref. [42],

$m=\rho_{0} \int_{0}^{r_{\mathrm{k}}} 2 \pi \frac{(\rho)}{\rho_{0}} r d_{\mathrm{g}} d r$

where $m$ is the mass of the kernel and $d_{\mathrm{g}}$ is the spark gap. The average temperature of this ideal gas kernel is

$T=\left[\frac{p V_{\mathrm{k}}}{\gamma-1}\right] /\left(m C_{\mathrm{v}}\right)=\frac{p W V_{\mathrm{k}}}{m Z}$,

where $V_{\mathrm{k}}=\pi r_{\mathrm{k}}^{2} d_{\mathrm{g}}, Z$ is the universal gas constant, and $W$ is the molecular weight. Because of the high temperature near the center of the blast up to this point in time, all the fuel in the kernel is assumed to be burned, increasing the size and temperature accordingly. The contribution of this chemical energy release is significant. This is evidenced in the prior comparison between spark kernels in air and a combustible mixture in Section II. The initial kernel having the above properties is a result of the blast wave generated by the high power energy input during the electrical breakdown of the gap. 


\section{Diffusion Model}

Kernel growth as a diffusion wave with an initial condition taken from the blast wave is modeled as a sphere as suggested by the experiment. The fresh mixture enters and immediately burns as in a steady laminar flame. The schlieren pictures suggest that there is also an inflow of unburned mixture entering the kernel along the electrodes while the spark is discharging. This discharge induced entrainment helps to explain the initially rapid kernel growth. The rate of mass increase is therefore expressed in two terms:

$\dot{m}=\frac{d}{d t}\left(\frac{4}{3} \pi R 3 \rho\right)=4 \pi R^{2} \rho_{\mathrm{u}} C_{\mathrm{s}} S_{\mathrm{l}}+\rho_{\mathrm{u}} C_{\mathrm{d}} u$.

$R$ and $\rho$ represent the kernel radius and density of the gas in the kernel while the subscript $u$ refers to the unburned fresh mixture. $S_{1}$ is the laminar burning speed and $C_{\mathrm{s}}$ and $C_{\mathrm{d}}$ are adjustable constants. The first term in this equation corresponds to pure diffusive burning along the kernel boundary with $C_{\mathrm{s}}$ being a ratio between the actual burning speed and the normal burning speed of a steady laminar flame. The second term simulates the discharge induced mass inflow along the electrodes. The dimensional constant $C_{\mathrm{d}}$ is the lumped product of an inflow area, which is assumed to be constant as a first approximation, and a proportionality constant between the actual inflow velocity and a computed entrainment velocity. The entrainment velocity $u$ is derived from the assumption that some unknown fraction of the input power $\dot{E}_{\mathrm{e}}$ is converted to a kinetic energy flux of the gas into the kernel at a quasi-steady condition as follows:

$\dot{E}_{\mathrm{e}} \approx\left(4 \pi R^{2}\right) \rho_{\mathrm{u}} u^{3}, \quad u \approx\left[\frac{\dot{E}_{\mathrm{e}}}{4 \pi R^{2} \rho_{\mathrm{u}}}\right]^{1 / 3}$.

Since the inflow area is very small compared to the units of the constant $\left(\mathrm{m}^{2}\right)$ and only a fraction of the input power is converted to a kinetic energy flux, the value of $C_{\mathrm{d}}$ resulting from their product is necessarily small.

An energy equation is formed from a balance of the rate of increase of internal energy, rate of expansion work, enthalpy flow across the bound- ary, spark power, and chemical energy generation:

$\frac{d h}{d t}=\frac{1}{\rho} \frac{d p}{d t}-\left(h-h_{\mathrm{u}}\right) \frac{\dot{m}}{m}+\frac{\dot{E}_{\mathrm{e}}}{m}+\dot{m} Q_{\mathrm{c}}$,

where $h$ is the enthalpy and the subscript $u$ refers to the unburned mixture. Since the effects of dissociation and ionization are not included in the model, the heat of combustion per unit mass of the mixture, $Q_{\mathrm{c}}$, is reduced until the predicted combustion temperature equals the adiabatic flame temperature from a JANAF computation. This reduction is dependent upon equivalence ratio and is on the order of $10-20 \%$ of the lower heating value.

The equation of state for an ideal gas,

$p=\frac{\rho Z T}{W}$,

completes the formulation, where $W$ is the molecular weight of the mixture. The growth of the spark kernel is predicted by numerically solving Eqs. (6), (8), and (9) for the three unknowns ( $p$, $T$, and $R$ ) with the initial condition taken from the blast wave solution. The time dependent spark power $\dot{E}_{\mathrm{e}}$ can either be assumed or taken from measurements. The properties of air are used for both the unburned mixture and the combustion products. The enthalpy of $\mathrm{N}_{2}$ and $\mathrm{O}_{2}$ is computed from the JANAF tables.

\section{Electrode Heat Transfer}

The thermal conductivity of a metal is greater than that of a gas by at least two orders of magnitude. Since a spark kernel is in contact with the electrodes throughout its developing stage, a measurable amount of energy can be lost from the kernel to the electrodes through conduction, convection, and chemical recombination at the surface. This is especially true with massive electrodes. Efforts are usually made in experimental studies of spark ignition to reduce these effects by installing small electrodes and using a gap that is wider than the quenching distance. There currently are no spark ignition models that include the 
effect of heat transfer to the electrodes. It is an important factor to be considered, however, because automotive spark plugs typically have electrodes whose dimensions are of the same order as the gap dimension.

In an investigation of electrode heat transfer, Roth et al. [43] compute the percentage of spark energy that remains in the gas after a short capacitive spark from the measured pressure or volume increase in a vessel. The maximum thermal energy in the gas is observed to occur immediately after the spark and increases with increasing gap width, decreasing thermal conductivity of the gas, and decreasing electrode diameter as one would expect.

Saggau [44] presents the effect of discharge type, total energy, gap separation, and electrode material on energy transfer to the gas. The glow discharge is shown to have the lowest efficiency which is hypothesized to be a result of its large cathode fall voltage. A breakdown discharge has the highest efficiency while increasing the applied energy with any discharge type results in decreasing efficiency values. The effects of increasing gap width and electrode diameter are similar to those shown by Roth et al. [43].

A higher spark energy requirement with a glow discharge versus an arc discharge causes Swett [4] to speculate that the portion of spark energy released in the cathode and anode falls is largely unavailable for ignition because of the falls being in close proximity (less than $1 \mu \mathrm{m}$ ) to the electrode surface. This hypothesis is further supported in a recent experimental study by Ziegler et al. [13]. The difference in the minimum ignition energy between a glow and an arc discharge is shown to vanish when only the spark energy in the positive column of the discharge is compared.

In the absence of a more elaborate quantification of the heat loss to the electrodes, the above idea of Swett [4] and Ziegler et al. [13] is adopted in the present model. The net power input to the spark kernel is taken as the total spark power minus the power in the electrode fall regions:

$\dot{E}_{\mathrm{e}}=I_{\mathrm{s}}\left(\Delta V_{\text {total }}-\Delta V_{\text {anode }}-\Delta V_{\text {cathode }}\right)$,

where $I_{s}$ is the measured secondary current and $\Delta V_{\text {total }}$ the measured voltage.
According to Cobine [45], the heat transmitted to the anode by both glow and arc discharges is generally equal to the anode fall plus that due to the heat of condensation of the electrons at the anode surface which is given by the thermionic work function of the electrode material. The anode fall voltage is comparable to the least ionization potential of the gas. The value for an oxygen atom (13.6) is chosen for this work.

The model for the cathode fall voltage of the glow discharge is similarly taken from Cobine [45]. The cathode fall is on the order of $200-300 \mathrm{~V}$ and results from the inefficient process of electron emission by positive ion bombardment. It increases nearly linearly with the work function of the cathode material. A value of 0.034 for the electron emission constant of the stainless steel surface under positive ion bombardment yields a reasonable fit to the measured fall voltage.

The cathode fall voltage of the arc discharge is generally due to combined field and thermionic emission [45]. In this experiment, the electrodes are initially at ambient temperature and the arc discharge, if present, occurs immediately after breakdown. As a result, the cathode mechanism is believed to be mostly due to field emission. The cathode fall is of the order of the least ionization potential of the gas or vapor in which the arc burns. This work assumes a vapor is present and uses a potential of $6.8 \mathrm{~V}$.

The arc and glow part of the spark discharge is found on the measured voltage waveform by identifying transition points that are on the order of the cathode fall voltage. The lower voltage regions adjacent to the transition points are assumed to be arc discharges. Appropriate portions of power in the fall regions are then subtracted from the measured total value to obtain the net available spark power for ignition.

\section{DISCUSSION OF MODEL PREDICTIONS}

\section{Effects of Spark Energy}

The model developed in Section III needs to be calibrated at one condition by adjusting the two constants $C_{\mathrm{s}}$ and $C_{\mathrm{d}}$ until the predicted kernel 
radius matches the experimentally determined curve. This is done with the measured kernel radius, breakdown energy, and spark energy curve for the case of the $2.0 \mathrm{~mJ}$ spark discussed in Section II. As shown in Fig. 6, a reasonable fit is achieved, including the starting point derived from the ideal blast wave solution. The constants determined at this near minimum ignition energy condition $\left(C_{\mathrm{s}}=0.55\right.$ and $\left.C_{\mathrm{d}}=0.8 \mathrm{E}-7 \mathrm{~m}^{2}\right)$ are used in the rest of the study. One conclusion is that $C_{\mathrm{d}}$, the constant for the discharge induced inflow term, has a pronounced effect during the early part when the spark power is high, while $C_{\mathrm{s}}$, the adjustment to the laminar burning speed, primarily affects the nearly constant slope at later time.

Also shown in Fig. 6 is the prediction of the flame kernel growth pattern with the discharge data from the 11.5 and $54.8 \mathrm{~mJ}$ sparks discussed in Section II. A qualitative agreement is achieved in both cases. The trend of increasing kernel size with additional spark energy is adequately predicted. Although the prediction is slightly lower during the first $600 \mu \mathrm{s}$ for the spark of $11.5 \mathrm{~mJ}$ and a little higher after $800 \mu$ s for the case of $54.8 \mathrm{~mJ}$, the overall agreement is within the statistical variation of the data. It is interesting to note that the kernel sizes for all the cases in Fig. 6 are adequately correlated with a single value for the constant $C_{s}$ despite the apparently different slopes of the experimental curves.

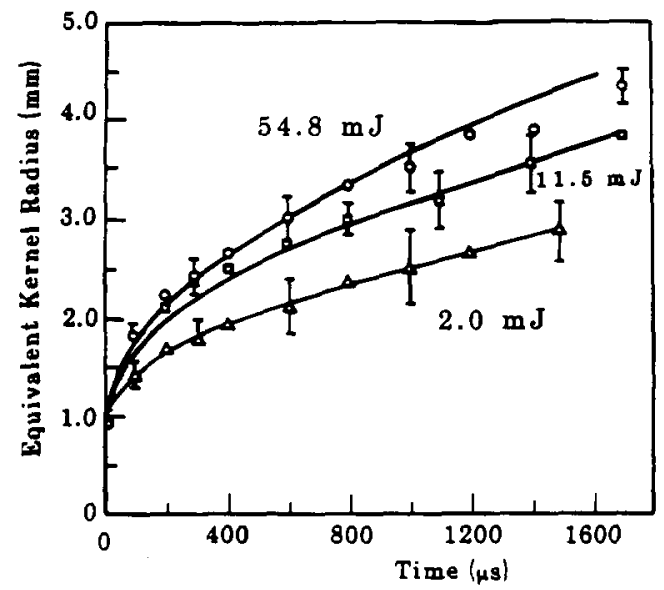

Fig. 6. Comparison of predicted (solid lines) and measured flame kernel radii for different spark energies at $\Phi=0.7$. The experimental data points are reproduced from Ref. [3].

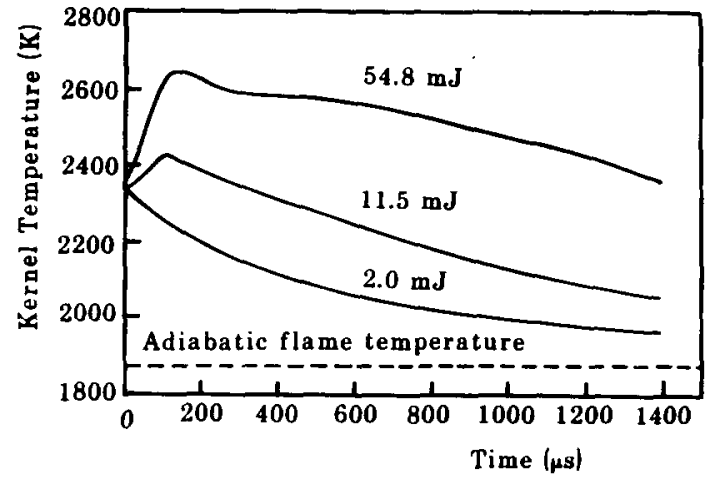

Fig. 7. Predicted kernel temperature for sparks of Fig. 6.

The average kernel temperatures from the model predictions are plotted in Fig. 7. The results indicate that increasing the spark energy raises the kernel temperature in addition to creating a larger kernel size through more mass entrainment. This higher temperature for a more energetic spark increases its expansion speed relative to that of a less energetic spark even though the burning speeds are identical.

When a nearly minimum amount of ignition energy $(2.0 \mathrm{~mJ})$ is released, the kernel temperature drops monotonically toward the adiabatic flame temperature of the mixture. An excess amount of spark energy over the minimum (11.5, $54.8 \mathrm{~mJ}$ ), however, initially raises the average temperature to a peak value, before decreasing again toward the adiabatic flame temperature. From a practical viewpoint, the hotter and larger flame kernel of an energetic spark will be less vulnerable to possible quenching under unfavorable circumstances. Such conditions can be manifested by a large metal surface near the spark gap, a small spark gap distance, and a high level of mixture motion.

Figure 8 shows that the fraction of the total energy in the kernel produced by the spark discharge generally decreases in time as one would expect. The percentage varies considerably in the time frame shown and depends upon the spark energy input. For the $54.8 \mathrm{~mJ}$ spark, more than $25 \%$ of the energy in the kernel is due to the spark after $1 \mathrm{~ms}$, while it is only about $10 \%$ or below in the other two cases. 


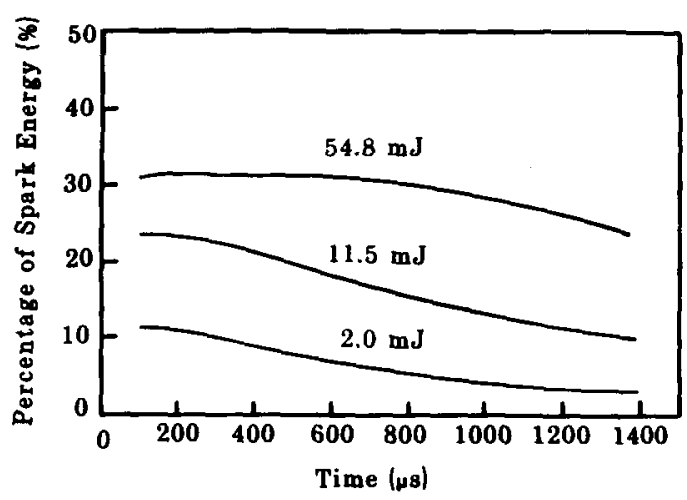

Fig. 8. Contribution of the spark to the total energy in the kernel for sparks of Fig. 6.

\section{Effects of Spark Power}

The model is applied to those sparks of the same energy but different power levels discussed in Section II. Experiments reveal that both sparks produce about the same size flame kernels. The model predictions are plotted in Fig. 9 along with the experimental data and also indicate that comparable flame kernels are generated. When compared more closely with the experimental radii, the predicted ones are slightly greater within the initial $100 \mu \mathrm{s}$. The kernel from a spark of high power level is predicted to have a slightly larger radius than that of a low power level $\left(I_{\mathrm{p}}=3.3 \mathrm{~A}\right)$ from 100 to $1000 \mu \mathrm{s}$, but ultimately assumes the

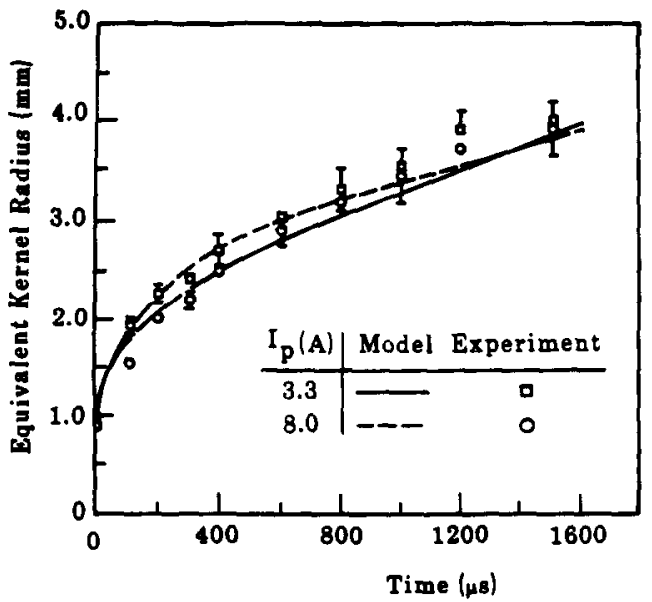

Fig. 9. Comparison of predicted and measured kernel radii for sparks of different power levels but of comparable total energy. The experimental power curves are shown in Fig. 4. same approximate size after $1200 \mu \mathrm{s}$. The fact that the high power spark has a larger predicted kernel radius during the electrical discharge is an expected result, but it is somewhat surprising that it ends up with the same size kernel as that of the spark of lower power but equal energy. Apparently the mass entrained during the longer discharge compensates the initially larger surface area in the higher power case, at least for the mixture and the power levels considered in this study.

\section{Effects of Equivalence Ratio}

A prediction of spark kernel development in a stoichiometric mixture is shown in Fig. 10 for a $12.4 \mathrm{~mJ}, 850 \mu$ s discharge. The agreement with experiment is quite reasonable although the model prediction slightly exceeds the experimental values. The difference, however, is within the accuracy of the experiment and becomes insignificant after approximately $1 \mathrm{~ms}$. The broken lines are model predictions for an identical spark in mixtures of equivalence ratios of 0.70 and 0.85 . The variation of the kernel size with mixture strength is

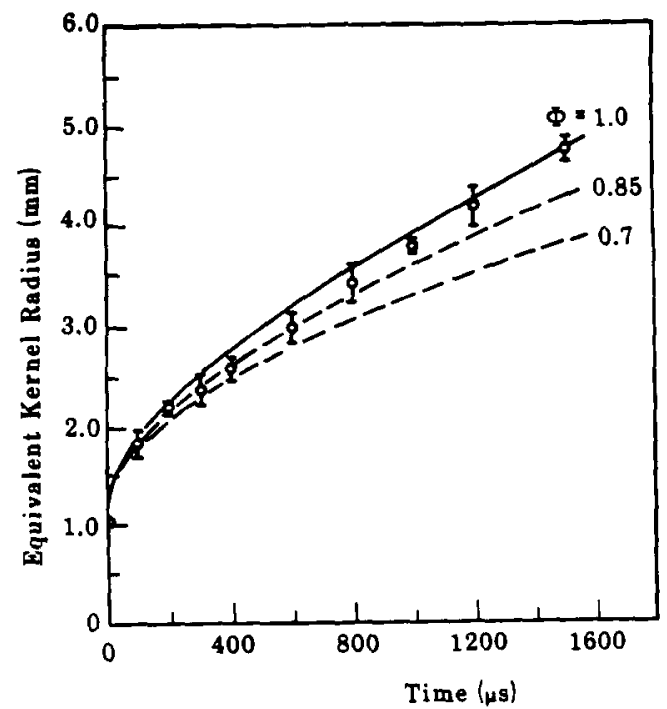

Fig. 10. Comparison of predicted (solid lines) and measured kernel radii in a propane-air mixture of $\Phi=1.0$. The broken lines represent calculations for different equivalence ratios with the same $12.4 \mathrm{~mJ}$ spark. 
reasonably similar to the experimental results of Fig. 5 which have a slightly lower spark energy of $11.5 \mathrm{~mJ}$ for the $\Phi=0.7$ case.

\section{SUMMARY AND CONCLUSIONS}

Flame kernel development from an inductive spark is investigated during the first 1-2 ms with a high speed $(10,000 \mathrm{fps})$ laser schlieren system. The measured equivalent kernel radii are presented for sparks of different energy and power in propane-air mixtures of different equivalence ratios. Simultaneous records of the released spark energy are recorded. Based upon experimental observations, a phenomenological model is developed to predict numerically the initial flame kernel growth. The major findings of the study are

1. Burning speed of the kernel, based on the expansion speed and the adiabatic flame temperature, attains an approximately constant value at about $1 \mathrm{~ms}$ which is less than the burning speed of a planar steady flame. Effects of flame curvature and the electrodes are most likely responsible for this low burning speed.

2. Kernel radii of equivalent sparks in air and propane-air mixtures exhibit a difference as early as $5 \mu$ s after breakdown. This suggests that kernel growth by chemical energy release occurs very early in the process.

3. The initially rapid growth of the kernel during the spark discharge can be adequately predicted with a mass entrainment term that is a function of the electrical power input.

4. Inductive sparks of different power levels (differing by a factor of about 2.5) but of comparable energy produce about the same size kernels within the time frame of the experimental data $(1500 \mu \mathrm{s})$. This suggests a relatively weak dependence of the kernel size on the rate of energy input in the current range of automotive engine use.

5. The phenomenological model reasonably predicts the effects of spark energy, spark power, and equivalence ratio on early flame kernel growth with two constants adjusted at one condition.

This phenomemological model forms a basis from which an engine ignition model can be developed. Before engine validation is attempted, however, the model requires modifications to account for the effects of heat transfer from the initial flame kernel to the electrodes, the transition to the laminar flame speed, and an ignition criterion. These studies can be adequately conducted in a bomb environment. The one remaining effect, turbulence, requires engine validation unless an adequate experimental means of simultaneously duplicating the correct scales and intensities becomes available.

\section{REFERENCES}

1. Quader, A. A., SAE paper $760760,1976$.

2. Dale, J. D., and Oppenheim, A. K., SAE paper 810146, 1981.

3. Lim, M. T., PhD Thesis, The University of Michigan, Ann Arbor, 1985.

4. Swett, C. C. Jr., NACA Tech. Rep. 1287:799-816 (1956).

5. Hattori, T., Goto, K., and Ohigashi, S., I. Mech. E. Conf. Pub. 1979-9, London, 1979, pp. 153-164.

6. Kono, M., and Hatori, K., Proceedings of the Eighth International Conference on Gas Discharges and Their Applications, Pergamon Press, 1985, pp. 500503.

7. Ballal, D. R., and Lefebvre, A. H., Combust. Flame 24:99-108 (1975).

8. Kono, M., Kumagai, S., and Sakai, T., Sixteenth Symposium (International) on Combustion, The Combustion Institute, Pittsburgh, 1977, pp. 757-766.

9. Kono, M., Kumagi, S., and Sakai, T., Combust. Flame 27:85-98 (1976).

10. Steiner, J. C., and Mirsky, W., SAE paper 660346 , 1966.

11. Albrecht, H., Bloss, W. H., Herden, W., Maly, R. Saggau, B., and Wagner, E., SAE paper 770853, 1977.

12. Maly, R., and Vogel, M., Seventeenth Symposium (International) on Combustion, The Combustion Institute, Pittsburgh, 1979, pp. 821-831.

13. Ziegler, G. F. W., Wagner, E. P., and Maly, R. R., Twentieth Symposium (International) on Combustion, The Combustion Institute, Pittsburgh, 1985, pp. 1055-1069.

14. Kumagai, S., and Sakai, T., Eleventh Symposium (International) on Combustion, The Combustion Institute, Pittsburgh, 1967, pp. 995-1001.

15. Olsen, H. L., Edmonson, R. B., and Gayhart, E. L., J. Appl. Phys. 23:1157-1162 (1952).

16. Olsen, H. L., Gayhart, E. L., and Edmonson, R. B. Fourth Symposium (International) on Combustion, Williams and Wilkins, 1953, pp. 144-148. 
17. Linton, D. R., and Wooding, E. R., Brit. J. Appl. Phys. 10: 159-166 (1959).

18. de Soete, G. G., Central States Meeting, The Combustion Institute, March, 1981.

19. de Soete, G. G., Proc. I. Mech. E. C59/83:93-100 (1983).

20. Dixon-Lewis, G., and Shepherd, I. G., Fifteenth Symposium (International) on Combustion, The Combustion Institute, Pittsburgh, 1975, pp. 1483-1491.

21. Rajan, S., Central States Meeting, The Combustion Institute, April, 1979.

22. Akindele, O. O., Bradley, D., Mak, P. W., and McMahon, M., Combust. Flame 47:129-155 (1982).

23. Sher, E., and Refael, S., Nineteenth Symposium (International) on Combustion, The Combustion Institute, Pittsburgh, 1982, pp. 251-257.

24. Refael, S., and Sher, E., Combust. Flame 59:17-30 (1985).

25. Sloane, T. M., Combust. Sci. Tech. 34:317-330 (1983).

26. Anderson, R. W., and Lim, M. T., Combust. Sci. Tech. 43:183-196 (1985).

27. Lichfield, E. L., Combust. Flame 5:235-241 (1961).

28. Chomiak, J., Seventeenth Symposium (International) on Combustion, The Combustion Institute, Pittsburgh, 1979, pp. 255-263.

29. de Soete, G. G., Thirteenth Symposium (International) on Combustion. The Combustion Institute, Pittsburgh, 1971, pp. 735-743.

30. Adelman, H. G., Eighteenth Symposium (International) on Combustion. The Combustion Institute, Pittsburgh, 1981, pp. 1333-1342.

31. Anderson, R. W., and Lim, M. T., Proceedings of the
Eighth International Conference on Gas Discharges and Their Applications, Pergamon Press, 1985, pp. 511-514.

32. Lavoie, G. A., SAE paper 780229, 1978.

33. Lewis. B., and von Elbe, G., J. Chem. Phys. 15:803808 (1947).

34. Fenn, J. B., Ind. Engrg. Chem. 43:2865-2869 (1951).

35. Ballal, D. R., and Lefebvre, A. H., Fifteenth Symposium (International) on Combustion, The Combustion Institute, Pittsburgh, 1974, pp. 1473-1481.

36. Ballal, D. R., and Lefebvre, A. H., Proc. Roy. Soc. London A357:163-181 (1977).

37. Maly, R., Eighteenth Symposium (International) on Combustion, The Combustion Institute, Pittsburgh, 1981, pp. 1747-1754.

38. Arici, O., Tabaczynski, R. J., and Arpaci, V. S., Combust. Sci. Tech. 30:31-45 (1983).

39. Taylor, G. I., Proc. Roy. Soc. London A201:159-174 (1950).

40. Taylor, G. I., Proc. Roy. Soc. London A201:175-186 (1950)

41. Sedov, L. I., Similarity and Dimensional Methods in Mechanics (M. Holt, Ed.) Academic Press, New York, 1957, p. 363.

42. Plooster, M. N., Phys. Fluids 13:2665-2675 (1970).

43. Roth, W., Guest, P. G., von Elbe, G., and Lewis, B., J. Chem. Phys. 19:1530-1535 (1951).

44. Saggau, B., Archiv Elektrotech. 64:229-235 (1981).

45. Cobine, J. D., Gaseous Conductors, Dover Publications, New York, 1958, pp. 216-226, 246-248, 300$311,343-344$.

Received 8 September 1986; revised 18 March 1987 